\title{
Cosmic Infrared Background from Early Epochs - Searching for Signatures of the First Stars
}

\author{
A. Kashlinsky \\ SSAI and Code 665, Goddard Space Flight Center, Greenbelt, MD 20771, U.S.A.
}

\begin{abstract}
Cosmic infrared background (CIB) contains emission from epochs inaccessible to current telescopic studies, such as the era of the first stars. We discuss theoretical expectations for the CIB contributions from the early population of massive stars. We then present the latest results from the ongoing project by our team [9, 10, 11, 12] to measure/constrain CIB fluctuations from early epochs using deep Spitzer data. The results show the existence of significant CIB fluctuations at the IRAC wavelengths (3.6 to $8 \mu \mathrm{m}$ ) which remain after removing galaxies down to very faint levels. These fluctuations must arise from populations that have a significant clustering component, but only low levels of the shot noise. Furthermore, there are no correlations between the source-subtracted IRAC maps and the corresponding fields observed with the HST ACS at optical wavelengths. Taken together, these data imply that 1 ) the sources producing the CIB fluctuations are individually faint with $S_{v}<$ a few nJy at 3.6 and $4.5 \mu \mathrm{m} ; 2$ ) are located within the first $0.7 \mathrm{Gyr}$ (unless these fluctuations can somehow be produced by - so far unobserved - local galaxies of extremely low luminosity and with the unusual for local populations clustering pattern), 3) they produce contribution to the net CIB flux of at least $1-2 \mathrm{nW} / \mathrm{m}^{2} / \mathrm{sr}$ at 3.6 and $4.5 \mu \mathrm{m}$ and must have mass-to-light ratio significantly below the present-day populations, and 4) they have angular density of $\sim$ a few per $\operatorname{arcsec}^{2}$ and are in the confusion of the present day instruments, but can be individually observable with JWST.
\end{abstract}

Keywords: Stars: formation; large-scale structure of the universe; early universe PACS: $97.20 . \mathrm{Wt}, 98.80-\mathrm{k}$

\section{Introduction}

Cosmic infrared background (CIB) contains emissions produced during the entire history of the Universe, including those arising from the epochs of the first stars (see [5] for review). Isolating the part of the CIB coming mostly from the first stars epochs would provide important information about the emissions, evolution and contents of this era. This paper reviews the recent measurements done by our team in deep Spitzer IRAC data (Kashlinsky, Arendt, Mather \& Moseley - herafter KAMM, [9, 10, 11, 12]) and their interpretation.

The paper is structured as follows: it starts with a brief discussion of the theoretical motivation for our measurements, then presents a summary of the most recent results at the IRAC Channels $1(3.6 \mu \mathrm{m})$ and $2(4.5$ $\mu \mathrm{m})$. Our most recent analysis [12] showed that in the fields observed at both IRAC and optical ACS HST bands there are no correlations between the source-subtracted IRAC and the ACS source catalog maps. The absence of these correlations would place the sources producing these fluctuations within the first $0.7 \mathrm{Gyr}$ unless they originate in the more local, but extremely low luminosity galaxies that somehow escaped ACS detection. Finally, we discuss the data requirements on the nature of the populations producing these fluctuations: they have to originate from very faint sources, located within the first Gyr of the Universe's evolution. These require them to have mass-to-light ratio significantly below the present- day stellar populations and their number density is such that they are well within the confusion of the present instruments.

\section{Theoretical expectations}

An early generation of massive stars may have produced a non-negligible contribution to the CIB at the near-IR wavelengths. The net amount of that contribution can account for the entire claimed CIB excess (see review [5] for summary of measurements up to 2005) over the contribution due to the observed galaxy counts between 1 and $4 \mu \mathrm{m}$ if only $\sim 2-4 \%$ of the baryons went through these early stars [6]. The energy spectrum of the resultant CIB would be cut off below the Lyman limit at the redshift of these stars $(\sim 1 \mu \mathrm{m})$ and the fraction of the baryons locked in them would decrease linearly with the observed CIB excess flux. The latter would have to be below $\sim 0.2-0.5 \%$ if the NIR CIB excess is as low as the NICMOS measurements [14] suggest.

The left panel of Fig. 11 shows a template of early star emission at $z=10$ from [13]. The IRAC filters are marked with shaded areas; the figure shows that, although the IRAC bands are not centered at the peak of the emission produced by Ly- $\alpha$, their wavelengths cover the region containing much of the cumulative emission. This emission is likely to be reprocessed by the surrounding gas resulting in the model-dependent uncertainty at 

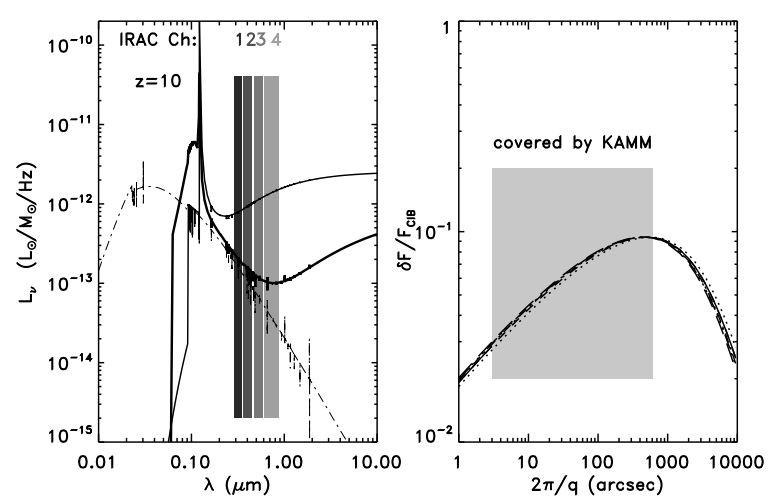

FIGURE 1. Left: SED from Pop III system is shown for $z=10$. The two solid lines are drawn from [13] for the case when re-processing of the radiation takes place in the gas inside the nebula and outside in the IGM. Thin dashed-dotted line shows the intrinsic SED prior to the emission re-processing by gas. Right: Lines show the fluctuations expected for concordance $\Lambda C D M$ model at $z=5$ to 20 for the parameters described in the text. Shaded area shows the angular scales probed by KAMM.

least as large as shown in the figure. Note that the color of the emission at IRAC channels, while approximately flat, can be either slowly or slowly decreasing with wavelength.

The first stars should also have left a distinct imprint in the CIB anisotropies, measuring which could give important information about that era and its constituents [1, 8]. There are intuitive reasons why the anisotropies can be significant: 1) first stars, if massive, emitted a factor $\sim 10^{5}$ more light per unit mass than the present-day stellar populations, 2) their relative fluctuations would be larger because they span a relatively narrow time-span in the evolution of the Universe, and 3) they formed (presumably) at the high peaks of the underlying density field, which would amplify their clustering properties.

The CIB fluctuations, $\delta F$, on scale $2 \pi / q$ are related to the 2-D power spectrum, $P_{2}(q)$, of the diffuse light via $\delta F \simeq\left[q^{2} P_{2}(q) / 2 \pi\right]^{1 / 2}$. They are related to the CIB produced by them, $F$, and the underlying 3 -D power spectrum of the sources' clustering, $P_{3}(k)$, via the Limber equation which approximates as $\delta F \simeq F \Delta\left(q d_{A}^{-1}(z)\right)$, where $\Delta^{2} \equiv \frac{1}{2 \pi^{2}} \frac{k^{2} P_{3}(k)}{\Delta t}$ is the mean square fluctuation in the source counts over a cylinder of radius $\sim k^{-1}$ and length $c \Delta t$ assuming the sources span cosmic time $\Delta t$. The right panel of Fig. 1 shows the typical relative fluctuation in the CIB produced by the early sources over $z$ from 6 to 20 assuming a (realistic) "toy"-model with the concordance $\Lambda \mathrm{CDM}$ initial conditions where the amplification due to biasing is $A \simeq 0.3(1+z) \sqrt{\Delta t(\mathrm{Gyr})}$. The figure shows the distinct peak at $\simeq 0.2-0.3$ degrees which, for CDM class of models, corresponds to the horizon at the matter-radiation equality redshifted to $z$. The

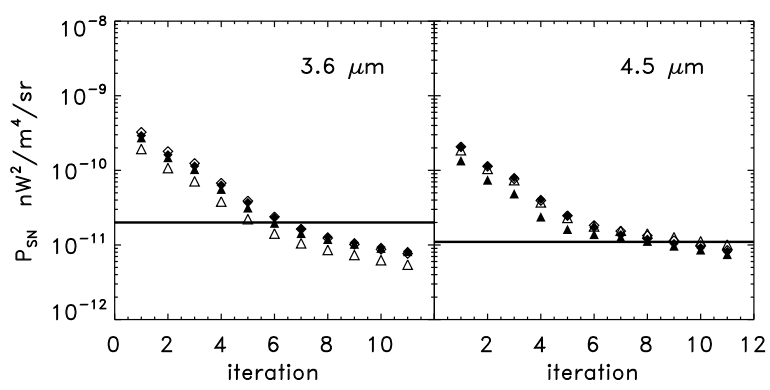

FIGURE 2. Shows decrease of the shot noise contribution to the power spectrum vs the iteration number of the KAMM source subtraction model. Horizontal lines show the levels reached in [10], which are a factor of $\sim 2$ below those in [9]. Triangles correspond to the HDFN region, diamonds to CDFS region; open symbols to Epoch 1, filled to Epoch 2.

shaded area shows the range of angular scales covered so far by the KAMM measurements. At these scales one can expect fluctuations of no more than $3-10 \%$, so the CIB fluctuation level of $\delta F \sim 0.1 \mathrm{nW} / \mathrm{m}^{2} / \mathrm{sr}$ would require CIB of at least $F \sim(1-2) \mathrm{nW} / \mathrm{m}^{2} / \mathrm{sr}$.

\section{CIB fluctuations from Spitzer deep images}

The KAMM methodology is summarized in [9, 10]. Briefly, the steps are: 1) The maps are assembled using the least calibration method of [2]. It solves for detector properties at the time of observations and for frame-toframe variations, and does not remove large-scale structure except due to (arbitrary) linear gradients. 2) The assembled IRAC maps are first clipped of resolved sources via an iterative procedure which removes all pixels (with a certain mask) with flux above a certain threshold. 3) The clipped maps are then further cleaned of the remaining emissions from resolved sources using an iterative variant of the clean procedure [4]. 4) The clipping is stopped so that enough pixels are left (in practice $>70 \%$ ) for robust computation of the fluctuations spectrum via Fourier transform. 5) When clipping is done to deeper levels, and progressively smaller fractions of pixels remain, we compute instead the correlation function, which is immune to masking [9]. 6) The uncertainties on the resultant power spectra (e.g. due to mask which destroys the strict orthogonality of the basis functions) are verified via simulations. 7) It is verified that, at the iterations used for measuring the final fluctuations, the subtracted sources components do not correlate with the residual diffuse emissions of the maps.

As of now we have analyzed results from five deep exposure regions in various parts of the sky [9, 10]. The latest results, shown here in Figs. 23, are from analysis of four independent regions mapped in the course of the 
GOODS project observations. They cover $\simeq 10^{\prime}$ on the side and have total exposure time of $\sim 23-25$ hours per pixel. The power spectrum of the diffuse emission in the source subtracted maps is made up of two components: 1) small scales are dominated by the shot-noise from the remaining sources, and 2) larger scales are dominated by the component of CIB fluctuations arising from clustering of the contributing sources. As we clean maps of progressively fainter sources, the shot-noise level decreases as shown in Fig. 2. At the same level of the shot noise all five regions exhibit the same large-scale fluctuations consistent with their cosmological origin. The figure also shows the shot-noise levels we stop at when presenting the results; they are limited by the floor imposed by the instrument noise levels. Comparing the shot-noise levels with those computed from the observed galaxy counts tells us that individual galaxies have been eliminated to $\mathrm{AB}$ magnitudes $>26$.

The upper panels in Fig. 3 show the CIB fluctuations from the GOODS data at 3.6 and $4.5 \mu \mathrm{m}$. Dotted lines show the shot-noise. We demonstrated that these fluctuations do not arise from the various Solar System and Galactic foregrounds and are not produced by the instrument systematics [9, 10]. The fluctuations also correlate well with maps at the longest IRAC channels (5.8 and $8 \mu \mathrm{m}$ ), although at these channels we do not recover CIB fluctuations with the same $\mathrm{S} / \mathrm{N}$ due to the larger instrument noise and possible cirrus pollution at $8 \mu \mathrm{m}$. The clustering component dominates fluctuations at $>20-30^{\prime}$. The solid line shows the fluctuation spectrum due to the concordance $\Lambda \mathrm{CDM}$ model at high $z$ assumed for simplicity to have the same amplitude at 3.6 and $4.5 \mu \mathrm{m}$. The figure shows that the signal is well described by the $\Lambda \mathrm{CDM}$ model with sources at high- $z$ and the color of these sources is approximately flat at the two IRAC bands.

\section{Cross-correlating NIR and optical data}

The GOODS fields have also been observed with the $H S T$ ACS instrument at optical wavelengths from $\sim 0.4$ to $0.9 \mu \mathrm{m}$ reaching source detection levels fainter than $28 \mathrm{AB}$ mag. If these fluctuations arise from local populations there should be a strong correlation between the source-subtracted IRAC maps and the ACS sources. Conversely, there should be no such correlations if the KAMM signal arises at at epochs where the Lyman break (at rest $\sim 0.1 \mu \mathrm{m}$ ) gets shifted passed the longest ACS z-band at $\simeq 0.9 \mu \mathrm{m}$. To test for this we [12] have constructed synthetic maps, overlapping with the GOODS fields, using sources in the ACS B, V,i,z bands from the ACS sources catalog [3]. These maps were then convolved with the IRAC 3.6 and $4.5 \mu \mathrm{m}$ beams. Finally,

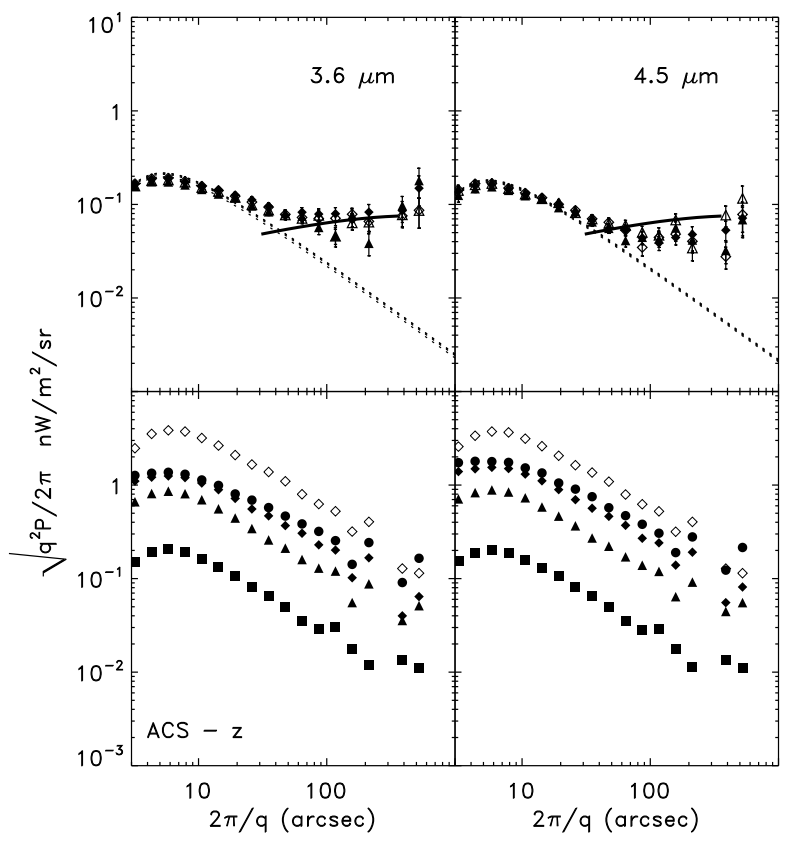

FIGURE 3. Top panels: CIB fluctuations from the sourcesubtracted maps from [12] at the shot-noise levels of [10]. Same symbol notation as in Fig. 2 Dotted lines show the shot-noise contribution. Solid line shows the slope of sources at high- $z$ with the $\Lambda \mathrm{CDM}$ model spectrum of the same amplitude at 3.6 and $4.5 \mu \mathrm{m}$. Lower panels: CIB fluctuations due to ACS galaxies for the $972 \times 9720.6^{\prime \prime}$ pixel field at HDFN-Epoch2 region for the ACS z-band. Right and left panels show the spectrum for the ACS source maps convolved with the IRAC 3.6 and 4.5 $\mu \mathrm{m}$ beams. Filled circles correspond to ACS galaxies fainter than $m_{0}=21$ with the mask defined by the clipping. Filled diamonds, triangles and squares correspond to fluctuations produced by sources fainter than $m_{0}+2, m_{0}+4, m_{0}+6$. Open diamonds show the fluctuations produced by galaxies fainter than $m_{\mathrm{AB}}=23$ when the clipping mask is not applied; the symbols show that such galaxies were effectively removed from the ACS maps by clipping alone done in KAMM.

we applied the clipping mask from the IRAC maps and computed the fluctuations spectrum produced by the ACS sources and their correlations with the IRAC-based maps.

The lower panels in Fig. 3 show the spectrum of fluctuations produced by the ACS z-band sources. It is clear from the figure that the ACS sources produce a completely different spectrum of the fluctuations than found by KAMM. This alone shows that they cannot produce the KAMM fluctuations signal shown in the upper panels. In fact, their power spectrum is very similar to that of the CIB fluctuations found in the deep 2MASS data, which arise from galaxies at $z>1$ [7]. Fig. 2e of ref. [7] shows the gradual evolution of the power spectrum 

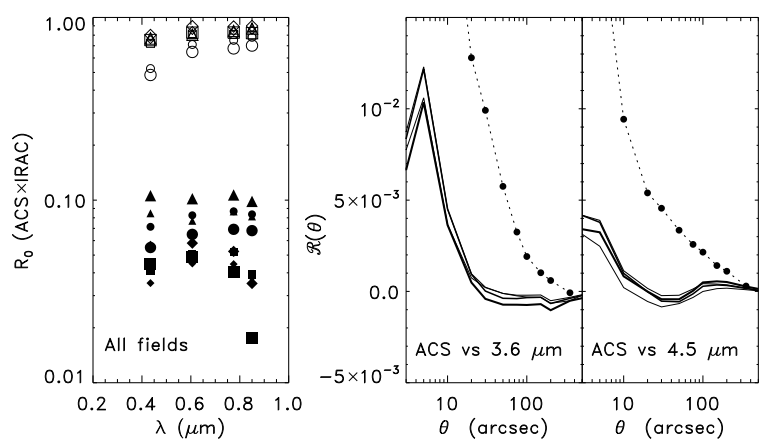

FIGURE 4. Left: Correlation coefficient between clipped/masked ACS and KAMM data. Large and small symbols correspond to the IRAC Ch 1 and Ch 2. Open symbols correspond to correlations with the maps of the removed sources and filled symbols with the residual KAMM maps. Circles, diamonds, triangles, squares correspond to HDFN-E1, HDFN-E2, CDFS-E2, CDFS-E2. Right: The various thickness solid lines show the dimensionless correlation function between the diffuse light in the ACS and KAMM maps for $B, V, i, z$-bands. Dotted line shows the dimensionless correlation function of the KAMM maps, $C_{\mathrm{KAMM}}(\theta) / \sigma_{\mathrm{KAMM}}^{2}$, which remains positive out to $\sim 100^{\prime \prime}$ and is better viewed when presented in log-log plots as in Fig. SI-4 of [9].

spectral slope towards deeper cut 2MASS data which is consistent with the evolution of the various slices of the ACS sources in the lower panels of Fig. 3 .

In ref. [12] we compute both the correlation coefficients, $R_{0}=\left\langle\delta F_{\mathrm{ACS}} \delta F_{\mathrm{KAMM}}\right\rangle / \sigma_{\mathrm{ACS}} \sigma_{\mathrm{KAMM}}$, and the full cross-correlation matrix, $C(\theta)=\left\langle\delta F_{\mathrm{ACS}}(\vec{x}) \delta F_{\mathrm{KAMM}}(\vec{x}+\right.$ $\vec{\theta})\rangle$ between the ACS and KAMM maps. In this representation, the contributions of any white noise (such as shot noise and/or instrument noise) component to $C(\theta)$ drop off very rapidly outside the beam and for the IRAC $3.6 \mu \mathrm{m}$ channel contribute negligibly to the correlation function at $\theta$ greater than a few arcsec. This is best seen from the analog of the correlation coefficient at non-zero lag, defined as $R(\theta) \equiv C(\theta) / \sigma_{\mathrm{ACS}} \sigma_{\mathrm{KAMM}}$. The mean square fluctuation on scale $\theta$ is given by the integral $\left\langle\delta F(\theta)^{2}\right\rangle=\frac{2}{\theta^{2}} \int_{0}^{\theta} C\left(\theta^{\prime}\right) \theta^{\prime} d \theta^{\prime}$. Hence, we evaluated from $R(\theta)$ a related quantity $\mathscr{R}(\theta)=\frac{2}{\theta^{2}} \int_{0}^{\theta} R\left(\theta^{\prime}\right) \theta^{\prime} d \theta^{\prime}$, shown in the right panels in Fig. 4 .

There are excellent correlations (shown with open symbols) between the ACS source maps and the sources removed by KAMM prior to computing the remaining CIB fluctuations in Fig. 3 . However, practically no correlations remain with the source-subtracted IRAC maps and the correlations are negligible outside the IRAC beam. This means that, at most, the remaining ACS sources contribute to the shot-noise levels in the residual KAMM maps (as discussed by [11]), but not to the large scale correlations in Fig. 3

\section{The nature of the sources.}

Any interpretation of the KAMM results must reproduce the following major aspects: $\bullet$ The sources in the KAMM data were removed to a certain (faint) flux limit, so the CIB fluctuations arise in populations with magnitudes fainter than the corresponding magnitude limit. - These sources must reproduce the excess CIB fluctuations by KAMM on scales $>0.5^{\prime}$, both their amplitude and their spatial spectrum. • The populations fainter than the above magnitude limit must account not only for the correlated part of the CIB, but - equally importantly they must reproduce the (low) shot-noise component of the KAMM signal, which dominates the power at $<0.5^{\prime}$. - The data show the absence of correlations between the source-subtracted IRAC maps and the same area mapped by ACS. • The CIB fluctuations signal is measured from the source-subtracted IRAC maps at all four channels (although not with the same $\mathrm{S} / \mathrm{N}$ at the longest wavelengths) and has color which is approximately flat with wavelength across the IRAC bands.

We briefly discuss the constraints in the above order:

1) Magnitude limits. The nominal limit above which sources have been removed in the KAMM analysis is $m_{\mathrm{AB}} \simeq 26$ at 3.6 and $4.5 \mu \mathrm{m}$ and this by itself implies that the detected CIB fluctuations arise from fainter systems. At this magnitude one is already at the confusion limit for the IRAC beam at $3.6 \mu \mathrm{m}$, so fainter galaxies can be excised only by removing significantly more pixels. This magnitude limit corresponds to $6 \times 10^{8} h^{-2} L_{\odot}$ emitted at $6000 \AA$ at $z=5$. A significant fraction of galaxies were thus removed from the data by KAMM even at $z \geq 5$ and the detected CIB fluctuations must be explained by still fainter and more distant systems.

2) Clustering component The relative CIB fluctuations produced by these sources are at most $10 \%$ (see Fig. 1 and [11]). Fig. 3 shows that the clustering strength at $\geq 1^{\prime}$ requires $\delta F_{\mathrm{CIB}} \sim 0.1 \mathrm{nW} / \mathrm{m}^{2} / \mathrm{sr}$. This meaning that the net CIB from sources contributing to the KAMM signal at $3.6 \mu \mathrm{m}$ is $>1-2 \mathrm{nW} / \mathrm{m}^{2} / \mathrm{sr}$.

3 ) Shot noise constraints. The amplitude of the shotnoise power gives a particularly strong indication of the epochs of the sources contributing to the KAMM signal [11]. This can be seen from the expressions for the shot noise $P_{\mathrm{SN}}=f(\bar{m}) F_{\text {tot }}\left(>m_{\mathrm{lim}}\right)$, where $f(m)$ is the flux of the source of magnitude $m$ and $F_{\text {tot }}(>m)$ is the net CIB flux produced by sources fainter than $m$ [5]. Fig. 3 shows the shot noise amplitude evaluated at 3.6 and $4.5 \mu \mathrm{m}$ is $P_{\mathrm{SN}} \simeq(2,1) \times 10^{-11} \mathrm{nW}^{2} / \mathrm{m}^{4} / \mathrm{sr}$. Above it was shown that the sources contributing to the fluctuations must have CIB flux greater than a few $\mathrm{nW} / \mathrm{m}^{2} / \mathrm{sr}$ and combining this with the values for $P_{\mathrm{SN}}$ shown leads to these sources having typical magnitudes $m_{\mathrm{AB}}>29-30$ or individual fluxes below a few nJy. 
4) No correlations with optical ACS data. Our results show that the source-subtracted CIB fluctuations in deep Spitzer images cannot originate in the optical galaxies detected in the GOODS ACS data. While the $B, V, i, z-$ band galaxies are well correlated with those seen and $r e-$ moved in the 3.6 and $4.5 \mu \mathrm{m}$ data, they correlate poorly with the the residual 3.6 and $4.5 \mu \mathrm{m}$ background emission. These galaxies also exhibit a very different spatial power spectrum than the KAMM maps and the amplitude of their fluctuations is generally also low. Whatever sources are responsible for the KAMM fluctuations, they are not present in the ACS catalog. Since the ACS galaxies do not contribute to the source-subtracted CIB fluctuations, the latter must arise at $z>7$ as is required by the Lyman break at rest $\sim 0.1 \mu \mathrm{m}$ getting redshifted past the ACS $z$-band of peak wavelength $\simeq 0.9 \mu \mathrm{m}$. This would place the sources producing the KAMM signal within the first 0.7 Gyr. If the KAMM signal were to originate in lower $z$ galaxies which escaped the ACS GOODS source catalog because they are below the catalog flux threshold, they would have to be extremely low-luminosity systems $\left(<2 \times 10^{7} h^{-2} L_{\odot}\right.$ at $\left.z=1\right)$ and these galaxies would also have to cluster very differently from their ACS counterparts as Fig. 3 shows.

5) Color. Color gives additional information on the nature of the sources producing these fluctuations. Fig. 3 shows that the clustering of the populations producing the KAMM signal at 3.6 and $4.5 \mu \mathrm{m}$ is well described by the concordance $\Lambda \mathrm{CDM}$ model with sources at high $z$ with the color approximately expected from populations described by SED of the type in Fig. 11 The signal is not as clear at the two longest IRAC bands, but the data at all four channels correlate meaning that the same sources contribute to all the wavelengths [9]. The color at shorter wavelengths can prove an interesting diagnostic [15], but requires additional assumptions on the epochs of the emissions (which determine the location of the Lyman break) and that the differently processed maps leave the same populations.

Additional information on the nature of the populations responsible for these CIB fluctuations, can be obtained from the fact that the significant amount of flux ( $>1-2 \mathrm{nW} / \mathrm{m}^{2} / \mathrm{sr}$ ) required to explain the amplitude of the fluctuations must be produced within the short time available at these high $z$ (cosmic times $<0.5-1 \mathrm{Gyr}$ ). This can be translated into the comoving luminosity density associated with these populations, which in turn can be translated into the fraction of baryons locked in these objects with the additional assumption of their $\Gamma \equiv M / L$ [11]. The smaller the value of $\Gamma$, the fewer baryons are required to explain the CIB fluctuations detected in the KAMM studies. It turns out that in order not to exceed the baryon fraction observed in stars, the populations producing these CIB fluctuations had to have $\Gamma$ much less than the solar value, typical of the present-day pop- ulations [11]. This is consistent with the general expectations of the first stars being very massive.

For a typical Population III system with an SED such as shown in Fig. 11 one expects $\Gamma \sim 10^{-3}-10^{-2} \Gamma \odot$ at the IRAC bands. Because the (low) shot-noise values at 3.6 and $4.5 \mu \mathrm{m}$ imply that the individual sources have flux below a few to $10 \mathrm{nJy}$, the bulk of these populations had to have only at most a few times $10^{5} M_{\odot}$ of stellar material if they are to explain the KAMM fluctuations [11]. Such sources would be below the detection threshold of the current high- $z$ Lyman-break searches.

Finally, in order to detect the faint sources responsible for the CIB fluctuations with fluxes below a few nJy embedded in the underlying sea of emissions, their individual flux must exceed the confusion limit [11]. If such sources were to contribute to the CIB required by KAMM data, at 3.6 and $4.5 \mu \mathrm{m}$ they had to have the average surface density of $\bar{n} \sim F_{\mathrm{CIB}}^{2} / P_{\mathrm{SN}} \sim 5 \operatorname{arcsec}^{-2}$. In order to avoid the confusion limit and resolve these sources individually at, say, 5-sigma level $(\alpha=5)$ one would need a beam of the area $\omega_{\text {beam }} \leq \alpha^{-2} / \bar{n} \sim 5 \times$ $10^{-3} \operatorname{arcsec}^{2}$ or of circular radius below $\sim 0.04$ arcsec. This is clearly not in the realm of the currently operated instruments, but the JWST could be able to resolve these objects given its sensitivity and resolution.

Acknowledgements I thank my collaborators, Rick Arendt, John Mather and Harvey Moseley for many contributions to the KAMM results, and the NSF AST0406587 grant for support.

\section{REFERENCES}

1. Cooray, A. et al 2004, Ap.J., 606, 611

2. Fixsen, D., Moseley, S.H. \& Arendt, R.G. 2000, Ap.J.Suppl., 128, 651

3. Giavilsco, M. et al 2004, Ap.J., 600, L93

4. Hogbom, J. 1974, A\&AS, 15, 417

5. Kashlinsky, A. 2004, Phys. Rep., 409, 361

6. Kashlinsky, A. 2005, Ap.J., 633 L5

7. Kashlinsky, A., Odenwald, S., Mather, J., Skrutskie, M. \& Cutri, R. 2002, Ap.J., 579, L53

8. Kashlinsky, A., Arendt, R.G., Gardner, J.P., Mather, J. \& Moseley, S.H. 2004, Ap.J., 608, 1

9. Kashlinsky, A., Arendt, R.G., Mather, J., \& Moseley, S.H. 2005, Nature, 438, 45

10. Kashlinsky, A., Arendt, R.G., Mather, J., \& Moseley, S.H. 2007, Ap.J., 654, L5

11. Kashlinsky, A., Arendt, R.G., Mather, J., \& Moseley, S.H. 2007, Ap.J., 654, L1

12. Kashlinsky, A., Arendt, R.G., Mather, J., \& Moseley, S.H. 2007, Ap.J., 666, L1

13. Santos, M., Bromm, V. \& Kaminowski, M. 2002, MNRAS, 336, 1082

14. Thompson, R., Eisenstein, D., Fan, X., Rieke, M. \& Kennicutt, R. 2007, Ap.J., 659, 667

15. Thompson, R., Eisenstein, D., Fan, X., Rieke, M. \& Kennicutt, R. 2007, Ap.J., in press. arXiv:0706.0547.v1[astro-ph] 